\title{
THE ALGORITHM FOR DYNAMIC OPTIMIZATION OF THE PRODUCTION CYCLE IN BEARING INDUSTRY
}

\author{
M.I. Geraskin, V.V. Egorova \\ Samara National Research University, Samara, Russia
}

\begin{abstract}
The authors consider the problems in production resources optimization planning by production cycle criteria inside the business process of industrial companies working in the interim manufacturing sectors. The authors have worked-out the models of static and dynamic order optimization for the company production resources in view of pricing and process restrictions. We have also developed the production cycle mechanism optimization to be used in simulating the optimum programs for the companies that work in Russian bearing industry.
\end{abstract}

Keywords: optimization model, optimum planning mechanism, production cycle, static model, dynamic model, net cost.

Citation: Geraskin MI, Egorova VV. The algorithm for dynamic optimization of the production cycle when custom planning in industry. CEUR Workshop Proceedings, 2016; 1638: 552-568. DOI: 10.18287/1613-0073-2015-1638-552568

\section{Introduction}

Industrial production is the determinant for the Russian national economy growth, specifying the industrial type of the national economy system, as within 2005-2014 the structural share of this parameter inside gross domestic product (GDP) was coming to around $63 \%$, dropping only during the period of $2008-2009$ crisis to $60-58$ $\%$ [17]. Respectively, the dynamic of industrial production pre-determines the GDP tendencies and as in shown in Fig. 1 is closely correlated with the rates of equipment and machine-building industry changes: 2005 - 2008 growth declining phase was followed by 2009 crisis, then the indices had restored their pre-crisis parameters and the period of 2010 - 2014 has become once again the continuation of recession tendency.

The bearing production industry being the basic sector of GDP industrial components, following the common trends during the considered period of time, demonstrates lesser acute fluctuations: during the periods of economic growth declining (2005 $2008)$ and recession $(2010-2014)$ the industry growth indices were lower that the indices for the other components, but in 2009 the declining in the industry has com- 
pensated the more deep fall-down in equipment and machine- building. The important role in Russian bearing production industry (around $13-17 \%$ in 2008 - 2014) $[15,16,17]$ was played by the companies located in Samara Region - Samara Bearing Plant (SBP) and Instrument Bearing Plant (IBP) and Fig. 2 illustrates the dynamics of production cycles ranging from 210 to 780 days. Respectively, for bearing production companies with their products being the assembly parts for equipment and machinebuilding, where the demand in these products is the derivative one, the most important problem is correlate with resource demand planning as based upon the minimization of production cycles.

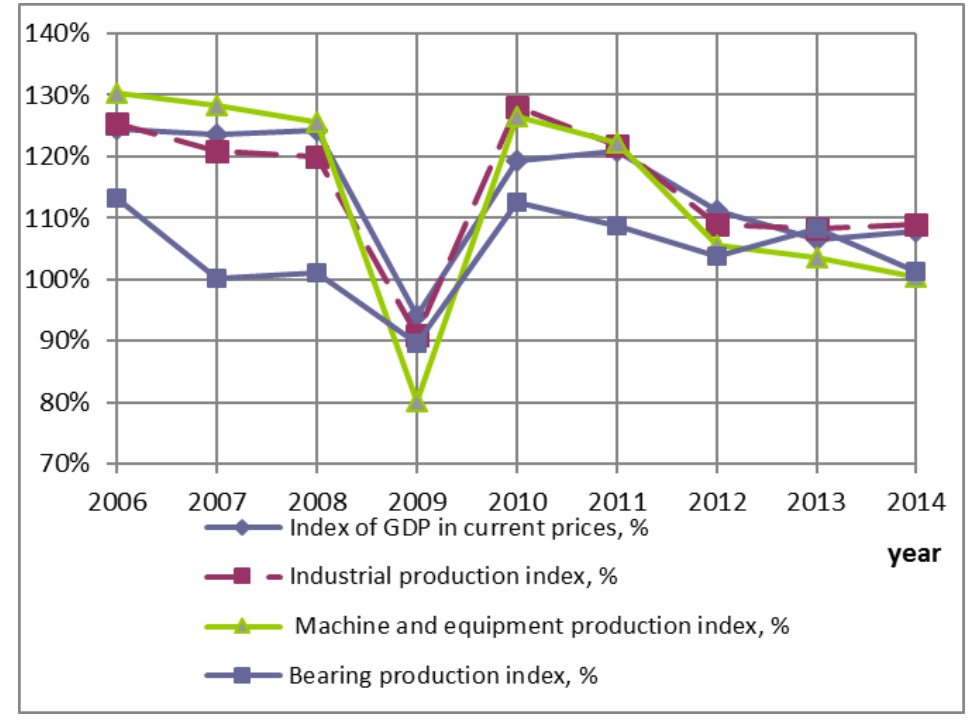

Fig. 1. Dynamic of 2006 - 2014 Russian GDP Industrial Components [17]

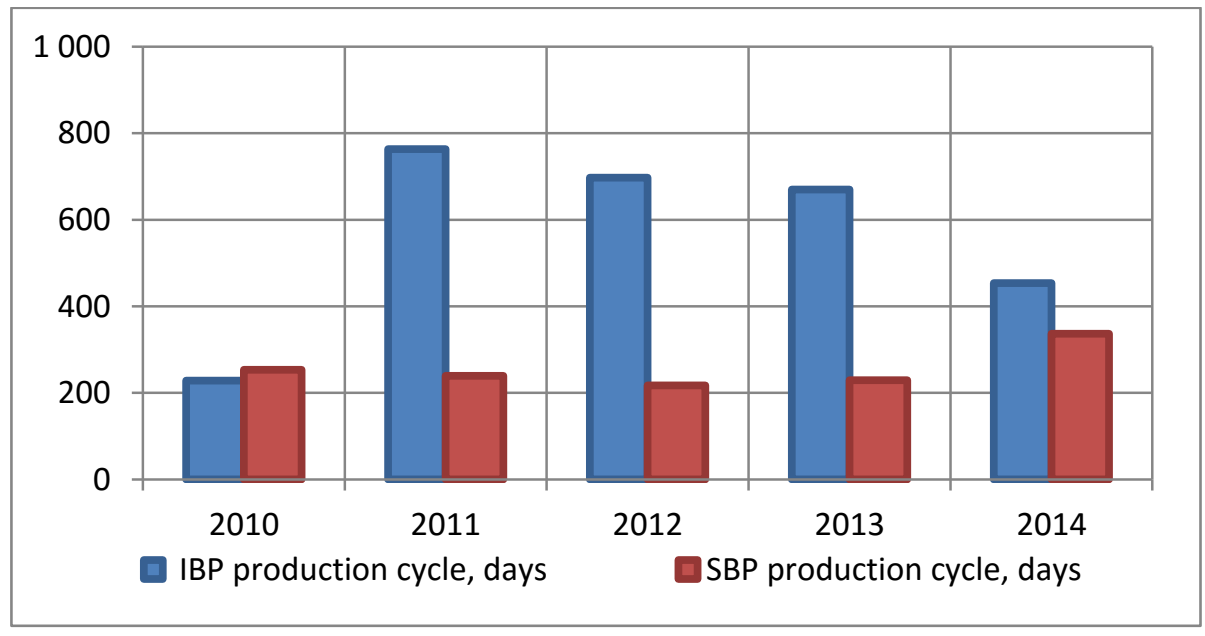

Fig. 2. $2010-2014$ SBP and IBP Production Cycles 
Within the frames of present-day studies the requirement on optimum planning as a rule if formed for one of the sub-processes in production Company business-plan (material provision, production or sales) with the restriction for the management parameters related to other sub-processes. As an example of optimality criteria we have considered the income in a form of resource linear function provided that temporary indices of the business-process were ignored [Ошибка! Источник ссылки не найден., 6, 8, 9, 10, 11, 14, 18, 19, 20, 23, 24]. Multi-process optimization models were shaped in a form of requirements with several criterion: we have considered the calendar planning with fuzzy restrictions [Ошибка! Источник ссылки не найден.,Ошибка! Источник ссылки не найден.] by income and cost criteria; we have optimized the nomenclature of produced products [Ошибка! Источник ссылки не найден.] be criteria of sales, income, net-cost and labour consumption and have optimized the projects from the view-points of timing and production cost criteria [13]. So, the present-day mechanisms of optimum production planning do not account for the availability of stable relations between the indices related various subprocesses of Company business-process as well as for the effect of timing factor in a form of production cycles in immobilization of the resources.

\section{Static Production Cycle Optimization Model}

Here we consider the task in optimum planning for the demand in Company businessprocess industrial resources as based upon the criterion of production cycle:

$$
\min F(u) \text {, }
$$

where, $F$-duration of Company production cycle. The matrix of managing parameters includes the scope of resource demand (listed requirements to the provision or payment) at various stages of industrial process

$$
u=\left\{u_{i}^{j}, i=1, \ldots, 3, j=1, \ldots, J_{i}\right\} .
$$

As for the resources we have studied such positions (having the index "i"), as materials, work-in-process (WIP), final products, nomenclature of which is marked by index "j".

Criterion of efficiency is determined as per the formulae (for the conclusion see Appendix):

$$
F(u)=\frac{T}{C(u)}\left(u_{\Sigma}^{0}+\frac{u_{1}+P(u)-C(u)}{2}\right), u_{\Sigma}^{0}=\sum_{i=1}^{3} u_{i}^{0}
$$

where, $\mathrm{T}$ - duration of the period (in days); $u_{i}^{0}, i=1, \ldots, 3$ - residual resources (materials, WIP and final products, respectively) at the beginning of the period; $\mathrm{C}$ - netcost of sales for the period; $\mathrm{P}$ - non-material industrial costs for the period (labour costs, social deductions, depreciation and other expenses). 
As the presentation of cycle (3) includes the explicit argument in a form of demands in material resources $u_{1}$ let's have a look at functions of non-productive c costs and expenses also through the dependence on this argument, and this will allow us to resolve the task in optimizing the functions using one variable value $u_{1}$. Foe this let's suppose the availability of stable interconnected trends in dynamics of demands for the Company products and scope of orders for resources at various stages of production as the function of material consumption during production $P\left(u_{1}\right), P_{u_{1}}^{\prime}\left(u_{1}\right)>0$ and material consumption for the final product manufacturing $C\left(u_{1}\right), C_{u_{1}}^{\prime}\left(u_{1}\right)>0$. Let's consider the following models of these trends in a form of power functions related to the interrelations between managerial parameters and status of the Company

$$
\begin{aligned}
& P\left(u_{1}\right)=B_{P} u_{1}^{\beta_{P}}, B_{P}>0,0<\beta_{P}<2, \\
& C\left(u_{1}\right)=B_{C} u_{1}^{\beta_{C}}, B_{C}>0,0<\beta_{C}<2,
\end{aligned}
$$

where, $B_{C}, B_{P}, \beta_{C}, \beta_{P}$ - factors of regressive models, restriction $0<\beta<2$ is put as related to real character of scale-expanding effect [Ошибка! Источник ссылки не найден.]. Note, that functions (4) - (5) may not reflect the direct regressive relations with argument $u_{1}$ and are formed by applying the most correlated components of matrix (2), and then the consistent result of the regressions as will be illustrated during the simulation stage.

Now, let's suppose the availability of prices declining trends for the resources, acquired by the Company, $z_{j_{1}}=z_{j_{1}}\left(u_{1 j_{1}}\right), j_{1}=1, \ldots, J_{1}, z_{j_{1} u}^{\prime}<0$, simulated in a form of power functions

$$
z_{j_{1}}\left(u_{1 j_{1}}\right)=A_{z j_{1}} u_{1}^{\alpha_{z j 1}}, \alpha_{z j_{1}}<0, j_{1}=1, \ldots, J_{1}
$$

where, $A_{z j_{1}}, \alpha_{z j_{1}}$ - factors of price regressive models for resources.

The following managerial restrictions are taken into consideration here. One is the restriction for the resource consumption norms versus the scope of Customer's demands and this is determined basing upon the consumption norms and residual resources:

$$
\begin{aligned}
& u_{1}^{\max }(N) \geq u_{1} \geq u_{1}^{\min }(N), \\
& u_{1 j_{3}}^{\max (\min )}=\sum_{j_{1}=1}^{J_{1}}\left[z_{j_{1}} \sum_{j_{3}=1}^{J_{3}} m_{j_{1} j_{3}}\left(N_{j_{3}}^{\max (\min )}-u_{3 j_{3}}^{0}-k_{j_{3}} \cdot u_{2 j_{3}}^{0}\right)\right],
\end{aligned}
$$

where $m_{j_{1} j_{3}}$ - mass consumption norm $j_{1}$ - resource for the production of 1 product item of $j_{3}$ type; $N_{j_{3}}^{\max (\min )}$ - demand fluctuation range for the product of $j_{3}$ type; 
$k_{j_{3}}$ - factor for the ready-made product of $j_{3}$ type from WIP; $z_{j_{1}}$ - procurement price for $j_{1}$ resource (per unit of the mass).

Restriction by the extreme level of procurement expenses

$u_{1} \geq u_{1}^{\min }(z)$,

is based upon price growth for the resources with the reduction of $u_{1}$ by trend (6).

Let's formulate the optimization demand for the resources using the criterion of production cycle

$u_{1}^{*}=\arg \min _{u_{1} \in U} F\left(u_{1}\right)$,

with due consideration of restrictions:

$U=\left\{u_{1} \in R^{+} \mid u_{1}^{\max }(N) \geq u_{1} \geq u_{1}^{\min }(N), u_{1} \geq u_{1}^{\min }(z)\right\}$,

where, $\mathrm{U}$ - is the area of permissible values for $u_{1}$.

\section{Mechanisms of Single-Period Cycle Optimization}

Let's find the boundary condition for the restriction (8) basing upon the minimum overall industrial expenses

$$
P_{\Sigma}(u)=\sum_{j_{1}=1}^{J_{1}} z_{j_{1}}\left(u_{1 j_{1}}\right) u_{1 j_{1}}+P\left(u_{1}\right)
$$

in a form of the following assertion proved in the Appendix.

Assertion 1: minimum of overall industrial expenses (11) at trends of types (4) and (6) is achieved for non-negative argument $u_{1 j_{1}}^{\text {min }} \geq 0$ in conditions

$$
\begin{aligned}
& u_{1 j_{1}}^{\min }(z)=\left(\frac{B_{P} \beta_{P}}{-\left(\alpha_{z j_{1}}+1\right) A_{z j_{1}}}\right)^{\frac{1}{\alpha_{z j}}}\left(\sum_{j_{1}=1}^{J_{1}} u_{1 j_{1}}^{\min }(z)\right)^{\frac{\beta_{P}-1}{\alpha_{z j_{1}}}}, \\
& j_{1}=1, \ldots, J_{1}, \\
& \beta_{P} \leq 1 \cap \alpha_{z j_{1}}<-1 \cap A_{z j_{1}}\left(\alpha_{z j_{1}}+1\right) \alpha_{z j_{1}} u_{1 j_{1}}^{\alpha_{z j_{1}}-1}>B_{P} \beta_{P} \mid \beta_{P}-1\left(\sum_{j_{1}=1}^{J_{1}} u_{1 j_{1}}\right)^{\beta_{P}-2} .
\end{aligned}
$$


The effective minimum for the overall industrial expenses (13) corresponds to the high-elastic pricing curves for the resources $\left(\alpha_{z j_{1}}<-1\right)$. As a rule, in life they implement the case with low-elasticity pricing curve $\left(\left|\alpha_{z j_{1}}\right|<1\right)$, and, respectively, the restriction (8) is of no importance, as the minimum in production expenses if reached at $u_{1 j_{1}}^{\min }<0$. Note that provision (13) is valid only as typical for the production companies at damped growth in non-material expenses together with the growth in resources demand ( $\beta_{P} \leq 1$ ).

Supposing that a Company is using a single-type material production resources it's possible to some extent to replace the actual factor of pricing coefficient and scope of resources demand by the average values (named as $\bar{\alpha}_{z}, \bar{A}_{z}, \bar{u}_{1}^{\min }$ ); thus, the equation (12) will have the analytical solutions as

$$
\bar{u}_{1}^{\min }(z)=\left(-\frac{B_{P} \beta_{P} J_{1}^{\beta_{P}-1}}{\left(\bar{\alpha}_{z}+1\right) \bar{A}_{z}}\right)^{\frac{1}{\bar{\alpha}_{z}-\beta_{P}-1}} .
$$

Let's formulate the mechanism of optimum planning for the criterion (1) of resource demand without any account $\mathrm{t}$ for the restrictions (10), presenting the optimum demand as $u_{1 F}^{*}$. Let's note preliminary that the joint analysis of trends (4) and (5) shows that the rate of growth for the non-material expenses and cost should satisfy the relation

$$
\beta_{P}<\beta_{C}+1
$$

as in other case $\left(\beta_{P}>\beta_{C}+1\right)$ the growth in non-materials resources should significantly overtake the growth in self-cost and this is impossible for the industry with high material consumption.

Assertion 2: minimum duration of production cycle (3) at trends (4) and (5) in view of (15) is achieved for non-negative argument $u_{1 F}^{*} \geq 0$, that satisfies the conditions as

$$
\begin{aligned}
& -2 u_{\Sigma}^{0} \beta_{c}+\left(1-\beta_{c}\right) u_{1 F}^{*}+B_{p}\left(\beta_{p}-\beta_{c}\right) u_{1 F}^{* \beta p}=0, \\
& u_{1 F}^{*} \in U_{1}=\left\{\beta_{C}>\beta_{P} \cup \beta_{C}<\beta_{P} \cap \beta_{C}+1>\beta_{P} \cap \varphi\left(\beta_{C}, \beta_{P}, u_{\Sigma}^{0}, u_{1}\right)>0\right\}, \\
& \text { where, } \begin{array}{l}
\varphi\left(\beta_{C}, \beta_{P}, u_{\Sigma}^{0}, u_{1}\right)=\beta_{C}\left[2\left(\beta_{C}+1\right) u_{\Sigma}^{0}+\beta_{C}-1\right] u_{1}+ \\
+B_{P}\left|\beta_{C}-\beta_{P}\right|\left(\beta_{C}+1-\beta_{P}\right) u_{1}^{\beta p} .
\end{array}
\end{aligned}
$$


In several specific and representative cases the equation (16) may be resolved analytically in a form of further mechanisms to optimize resource demand by criterion of production cycle duration, area of applicability of which are determined in view of $u_{1 F}^{*} \geq 0$ :

$$
\begin{aligned}
& u_{1 F}^{*}=\left(\frac{2 u_{\Sigma}^{0} \beta_{C}}{B_{P}\left(\beta_{P}-\beta_{C}\right)}\right)^{1 / \beta_{p}} \forall \beta_{C} \approx 1, \text { at }, \beta_{P}>\beta_{C} \\
& u_{1 F}^{*}=\frac{2 u_{\Sigma}^{0} \beta_{C}}{1-\beta_{C}} \forall \beta_{C} \approx \beta_{P}, \text { at }, \beta_{C}<1 \\
& u_{1 F}^{*}=\frac{2 u_{\Sigma}^{0} \beta_{C}}{1-\beta_{C}+B_{P}\left(\beta_{P}-\beta_{C}\right)} \forall \beta_{P} \approx 1, \text { at }, \beta_{C}<\frac{1+B_{P} \beta_{P}}{1+B_{P}} \\
& u_{1 F}^{*}=\frac{2 u_{\Sigma}^{0} \beta_{C}-B_{P}\left(\beta_{P}-\beta_{C}\right)}{1-\beta_{C}} \forall \beta_{P}<<1, \text { at } \beta_{C}<1 .
\end{aligned}
$$

Mechanism (18) that area of applicability for which correspond to the overtaking growth in non-material resources being higher than overall expenses $\left(\beta_{P}>\beta_{C}\right)$ is a result of direct proportionality conditions for product self-cost and demand in resources $\left(\beta_{C} \approx 1\right.$ ), that presents the complete use of material resources during the production phase. Having such a system of planning there is not accumulation in resources and there is no shortage in them as well. Mechanism (19) used in case for the damped growth in costs when compared to demand in resources $\left(\beta_{C}<1\right)$ presents the match between the rate of growth in non-material production costs and rate of growth in self-cost $\left(\beta_{P} \approx \beta_{C}\right)$ and this is realized within the conditions of rigid process regulations that ensure the constant co-relation of overall expenses and nonmaterial expenses and is typical for the system of normative planning (standard costing). Mechanism (20) is applied as a rule in case with $\beta_{C}<1$, as usually $\beta_{P}<1$, and this correspond to the concept of normative planning as in option (19), but in condition of establishing a constant correlation between demands in material resources and the level of non-material expenses ( $\beta_{P} \approx 1$ ). Finally, option (21) is typical for the Companies the production process of which has high material consumption (metallurgy, machine-building, metal-working, construction material industry). As a result of this the rate of growth in non-material expenses is significantly lower than the rate of growth for material resources $\left(\beta_{P}<<1\right)$. Area of applicability for mechanism (21) also correspond to $\beta_{C}<1$. So, the analytical mechanisms (18) - (21) are 
applicable to the most characteristic way in arranging the production process by leading production companies when due to process effect the rate of expenses growth is lower than the rate of material resources growth.

Analysis of mechanisms (18) - (21) shows that the value of $u_{1 F}^{*}$ in it order of magnitudes, is close to $u_{\Sigma}^{0}$, and as $u_{\Sigma}^{0}>>u_{1}^{0}$, then the cycle optimum is valid at the values that significantly exceed the average balance in material resources.

Note that in view of the correlation between order of magnitudes for the balance in reserves $u_{\Sigma}^{0}$, scope of demands $u_{1 F 1}^{*}$ and regression coefficients $\beta_{C}, \beta_{P}$ the following correlation is valid

$\beta_{C}\left[2\left(\beta_{C}+1\right) u_{\Sigma}^{0}+\beta_{C}-1\right] u_{1}>>B_{P}\left|\beta_{C}-\beta_{P}\right|\left(\beta_{C}+1-\beta_{P}\right) u_{1}^{\beta p}$, so the sufficient criterion of condition (17) is valid practically for all $u_{1 F 1}^{*} \geq 0$, therefore

$$
\varphi\left(\beta_{C}, \beta_{P}, u_{\Sigma}^{0}, u_{1}\right)>0 \forall u_{1 F 1}^{*} \geq 0
$$

Let's form the mechanism of resource demand optimum planning by criterion being the solution of provision (9) in a form of the following assertion.

Assertion 3: mechanism

$$
u_{1 F}^{*}=\max \left\{\min \left\{u_{1}^{*}, u_{1}^{\min }(N), u_{1}^{\min }(z)\right\}, u_{1}^{\max }(N)\right\}
$$

is the solution for the optimization task (9) with restriction (10).

Mechanism (23) is economically interpreted the following way: optimum of production cycle is achieved at such an index of demand in resources, that is the highest taken from the optimum value of demand by mechanism (16), is this is not coming outside the lower boundary of the demand (10) and maximum value of the demand (10) following the production schedule.

\section{Dynamic Model and Cycle Optimization Mechanisms}

Dynamics of Company production process is determined by the monthly or quarterly revisions of the plan due to the new demands from the Customers and this gives the growth in scope of demands and provides for the accumulation of material balance. So, the dynamic model of the production cycle is made in additive form by subperiods (by months or quarters) of period " $\mathrm{T}$ "

$$
F(u)=\sum_{t=1}^{\tau} \frac{T_{t} u_{\Sigma t}^{0}}{C_{t}(u)}+\sum_{t=1}^{\tau} \frac{T_{t}\left(u_{1 t}+P_{t}(u)-C_{t}(u)\right)}{2 C_{t}(u)}
$$

with the following recurrent correlations that are analogous to formula (P3), and determine the dependence of resource balance in subsequent sub-period on dynamics of procurement and production for the previous sub-period of period " $\mathrm{T}$ ": 
$u_{1}^{0(t+1)}=u_{1}^{0 t}+u_{1}^{t}-u_{2}^{t}, u_{2}^{0(t+1)}=u_{2}^{0 t}+u_{2}^{t}-u_{3}^{t}+P_{t}, u_{3}^{0(t+1)}=u_{3}^{0 t}+u_{3}^{t}-C_{t}$

where $u_{i}^{t}$ - scope of demand in resources in "t" sub-period of period " $\mathrm{T}$ "; $u_{\Sigma t}^{0}-$ the balance in resources at the beginning of pf " $\mathrm{t}$ " sub-period; $\tau$ - number of sub-periods in period; $\mathrm{T} \tau$-duration of sub-period.

Let's determine the dynamic correlations between the processes of production, manufacturing and product sales with the scope of demands and level of initial balance in resources in a form of power functions, analogous to (4), (5):

$$
\begin{aligned}
& P_{t}\left(u_{1 t}\right)=B_{P} u_{10 t}^{\beta_{P 0}} u_{1 t}^{\beta_{P}}, B_{P}>0,0<\beta_{P}<2,0<\beta_{P 0}<2, \\
& C_{t}\left(u_{1 t}\right)=B_{C} u_{10 t}^{\beta_{C 0}} u_{1 t}^{\beta_{C}}, B_{C}>0,0<\beta_{C}<2,0<\beta_{C 0}<2 .
\end{aligned}
$$

Substituting (26), (27) in (24) we get the expression of a cycle in " $t$ " sub-period:

$$
F_{t}(u)=\frac{T_{t} u_{\Sigma t}^{0}}{B_{C} u_{10 t}^{\beta_{C 0}} u_{1 t}^{\beta_{C}}}+\frac{T_{t}\left(u_{1 t}+B_{P} u_{10 t}^{\beta_{P 0}} u_{1 t}^{\beta_{P}}-B_{C} u_{10 t}^{\beta_{C 0}} u_{1 t}^{\beta_{C}}\right)}{2 B_{C} u_{10 t}^{\beta_{C 0}} u_{1 t}^{\beta_{C}}} .
$$

The arrangement of a discrete temporary line of managing parameters $u_{i}^{t}, \mathrm{t}=1, \ldots, \tau$ is supposed in the dynamic model of production cycle optimization. It is based on the minimization of criterion (28) in each sub-period of period " $\mathrm{T}$ " in compliance with the following dynamic restrictions:

$$
U_{t}=\left\{u_{1 t} \in R^{+} \mid u_{1 t}^{\max }\left(N_{t}\right) \geq u_{1 t} \geq u_{1 t}^{\min }\left(N_{t}\right), u_{1 t} \geq u_{1 t}^{\min }\left(z_{t}\right)\right\},
$$

where $U_{t}$ - the area of allowable values $u_{1 t}$ in " $\mathrm{t}$ " sub-period.

Let's formulate the production cycle optimization mechanisms (28) without the restrictions (29) and in view of these restrictions in a form of following assertions the evidence of which is not presented as they are analogous to assertions 2 and 3.

Assertion 4: minimum duration of production cycle (28) in " $\mathrm{t}$ " sub-period at trends (26) and (27) in view of (15) are achieved for the non-negative argument $u_{1 t}^{*} \geq 0$, that satisfies the conditions

$$
\begin{aligned}
& -2 u_{\Sigma t}^{0} \beta_{c}+\left(1-\beta_{c}\right) u_{1 t}^{*}+B_{p} u_{10 t}^{\beta_{P 0}}\left(\beta_{p}-\beta_{c}\right) u_{1 t}^{* \beta p}=0 \\
& u_{1 t}^{*} \in U_{1 t}=\left\{\beta_{C}>\beta_{P} \cup \beta_{C}<\beta_{P} \cap \beta_{C}+1>\beta_{P} \cap \varphi\left(\beta_{C}, \beta_{P}, u_{\sum t}^{0}, u_{1 t}\right)>0\right\},
\end{aligned}
$$


where $\varphi\left(\beta_{C}, \beta_{P}, u_{\Sigma t}^{0}, u_{1 t}\right)=\beta_{C}\left[2\left(\beta_{C}+1\right) u_{\Sigma t}^{0}+\beta_{C}-1\right] u_{1 t}+$ $+B_{P} u_{10 t}^{\beta_{P 0}}\left|\beta_{C}-\beta_{P}\right|\left(\beta_{C}+1-\beta_{P}\right) u_{1 t}^{\beta p}$

Assertion 5: mechanism $u_{1 F t}^{*}=\max \left\{\min \left\{u_{1 t}^{*}, u_{1 t}^{\min }\left(N_{t}\right), u_{1 t}^{\min }\left(z_{t}\right)\right\}, u_{1 t}^{\max }\left(N_{t}\right)\right\}$

is the solution of optimization task (28) with restriction (29).

\section{Production Cycle Dynamic Modeling}

Let's formulate the models with non-material expenses and self-costs (4), (5) and (27), (27) at the basis of data on quarterly dynamics related to the respective parameters of OAO "Samara Bearing Plant" and OAO "Instrument Bearing Plant" for 2011 - 2014 period through the application of the least square method algorithms:

$P\left(u_{1}\right)=8585 u_{1}^{0.3}, C\left(u_{1}\right)=45 u_{1}^{0.76}, P\left(u_{1 t}\right)=92.6 u_{10}^{0.08} u_{1}^{0.56}$,

$$
C_{t}\left(u_{1 t}\right)=0.6 u_{1 o}^{0.27} u_{1}^{0.76}
$$

The analysis of dynamic price statistical series and basic materials scope of supply used by the Companies, have verified the validity of the hypothesis (6) on declining price trends and have enabled us to formulate the single-phase regressions as follows:

$$
z_{1}\left(u_{1}^{1}\right)=205 u_{1}^{-0,3}, z_{2}\left(u_{1}^{2}\right)=105 u_{1}^{-0,2}
$$

The resulted regressions are adequate and verified in accordance with the determination factor evaluation that exceeds 0.93 and Fisher's ration test that is significantly higher than the critical value at $5 \%$ for the significance level.

Figure 3 presents the curves for the production cycle $F\left(u_{1}\right)$ that is calculated as per formulae (3) in view of regressions (33). We have also noted the restriction (7) in a form of $u_{1}^{\min }(N)$ and restriction (8) is not shown as the low-elastic pricing tendencies for the resources have been seen at the market (34) at which due to condition (13) the given restriction makes no difference. We show the actual values of the criterion valid for 2015. As per the mechanism (23) the solution of tasks (9) and (10) is the value of $u_{1}=u_{1}{ }^{\min }(N)$ at which the duration of cycle is lower than the actual data for 2015. The gross economic effect during profit immobilization in resources may come to RUB 5.8 million.

Dynamic optimization of production cycle is made as per the following sequence. At the basis of data on residual reserves at the beginning of the period (equal to respective dead-stock by the end of the previous period), as well as the predict for the scope of demand during " $\mathrm{t}$ " sub-period (at $\mathrm{t}=1, \ldots, \tau)$ they calculate the boundary values for the restriction (29) and define the optimum value of the demand by mechanism (32). 
Further they make the evaluation of resource balance by the end of " $\mathrm{t}$ " sub-period as per formula (25), after that they make another calculation of scope for the demands in $(\mathrm{t}+1)$ sub-period.

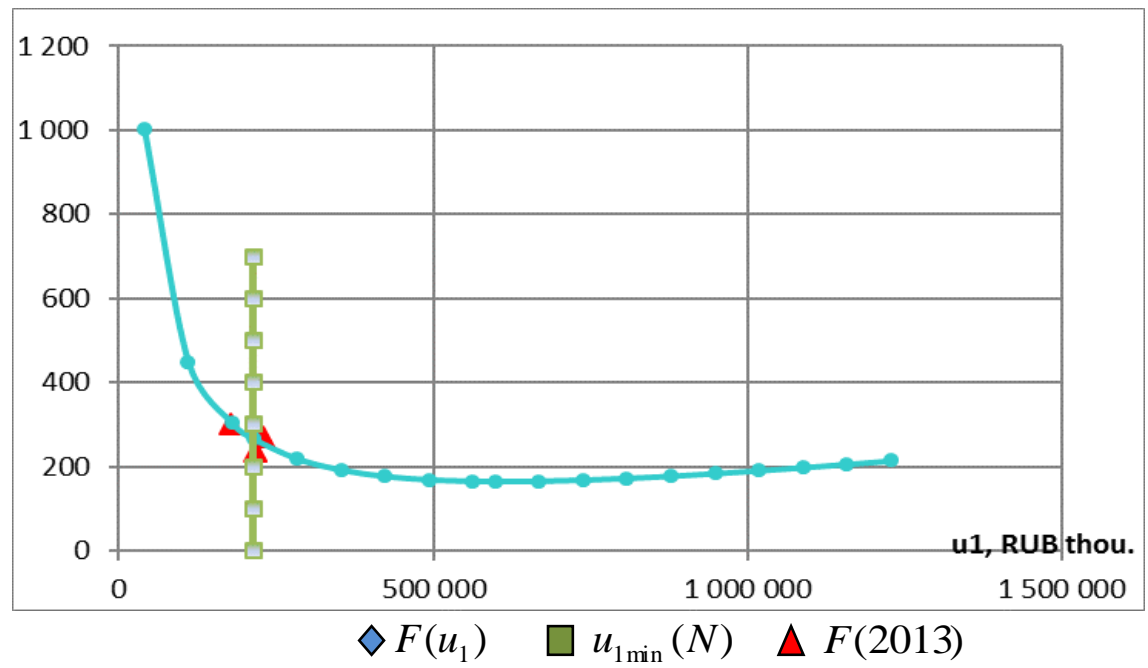

Fig. 3. Calculated and actual (in scope of 2015 demand) the values of production cycle (in days), restriction by the norms in resource consumption as dependent on the scope of Customer's demand (in thousands of RUBs)

Table 1. Results of Dynamic Optimization of 2015 Production Cycles at OAO "Samara Bearing Plant (in RUB thousands)

\begin{tabular}{llllllllll}
\hline $\mathbf{t}$ & $u_{1 t}^{0}$ & $u_{2 t}^{0}$ & $u_{3 t}^{0}$ & $u_{1 t}$ & $u_{2 t}$ & $u_{3 t}$ & $P_{t}(u)$ & $C_{t}(u)$ & $\begin{array}{c}F_{t}(u), \\
\text { days }\end{array}$ \\
\hline $\mathbf{1}$ & 120 & 57 & 156 & 41 & 51 & 121 & 71 & 118 & 54 \\
& 003 & 057 & 440 & 155 & 443 & 572 & 681 & 750 & \\
$\mathbf{2}$ & 109 & 58 & 159 & 38 & 48 & 113 & 66 & 122 & 56 \\
& 715 & 609 & 262 & 514 & 142 & 379 & 219 & 859 & \\
$\mathbf{3}$ & 100 & 59 & 149 & 35 & 44 & 91 & 72 & 113 & 69 \\
& 086 & 591 & 782 & 461 & 327 & 754 & 645 & 517 & \\
$\mathbf{4}$ & 91 & 84 & 128 & 40 & 50 & 106 & 73 & 117 & 67 \\
\multicolumn{7}{l}{ Actual duration of production cycle for 2015 } \\
\multicolumn{7}{l}{ Duration of production cycle for 2015 as per dynamic model } & 246 \\
\hline
\end{tabular}

Optimization results (Table 1) illustrate the reduction in production cycle duration resulted through the decrease in scope of demand and respective decrease in resource balance by quarters of the year, as well as the total value for the years from 286 to 246 days. By comparing the results of 2015 plan static modeling the duration of the cycle 
at dynamic modeling is reduced by 34 days and this illustrates the importance accounting to the dynamic factors while arranging the plans for the industrial demands.

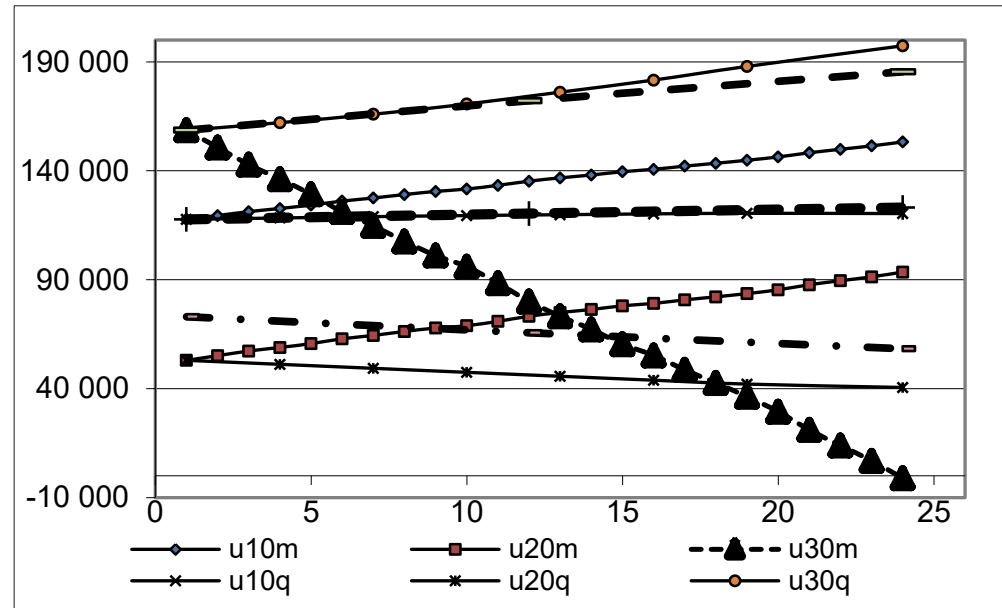

Fig. 4. Dynamics of raw materials, work in progress and finished products balances for 20152016 (ths. RUB)

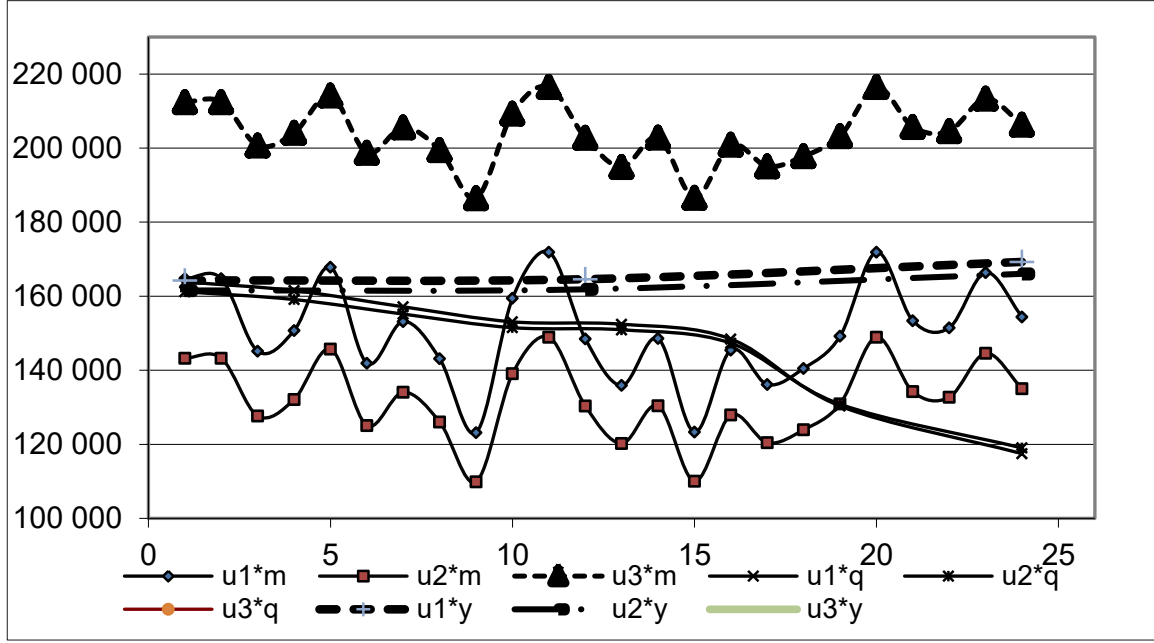

Fig. 5. Dynamics of orders by the types of resources for 2015-2016 (ths. RUB)

Optimization interval discretization analysis is given on the fig. 3 and 4, which shows the dynamics of aggregated balances and orders by the types of resources (2) for three variants optimal planning problem solving by the mechanism (23) for the planning period of 2015-2016: 1) optimization interval corresponds to the calendar year, designation " $y$ "; 2) optimization interval corresponds to the calendar quarter, designation "q"; 3) optimization interval corresponds to the calendar month, designation " $m$ ". In all three cases, there is a tendency of materials balances accumulation. This accumulation results from the planned production volume reduction. Work in progress balances 
go down in case of annual and quarterly planning due to the higher-than-anticipated planned production output growth. However, such balances go up in case of monthly planning that allows to take account of intraquarter distribution of output. Unsold output balances go up in case of annual and quarterly optimization. However, they go down in case of monthly planning, because it takes account of the sales intraquarter distribution; the latter aspect is the major contributor to the cycle time reduction in case of monthly planning in comparison with the first two variants. Comparison of cycle time values obtained as the result of optimization by three variants of discretization, with actual cycle values for 2015-2016, confirms that the planning interval reduction leads to the efficiency criterion increase.

\section{Conclusion}

We have designed the models of static and dynamic modeling for the industrial Company production cycles in view of the restrictions related to the Customer scope of demand and pricing police of material providers.

Static single-period model enables us to evaluate the scope of reserve acquisition that optimize the production cycle but is not taking into account the fluctuations in the production schedule being the result of irregularity in Customer scope of demand during a specific period of time (one year), thus reflecting the fluctuations in residual resources and substantiating the necessity in designing the dynamic model. The static model based upon the supposition expressing the dependency of production costs on dynamics of residual resources, is grounded on the predictions for the Company production costs versus the scope of demand in resources as per the historical information and is adequate with the stable level of resources balance in dynamics.

Dynamic model of the production cycle is based upon the prediction for the industrial costs and their dependence versus fluctuations in demand and balance in reserves. With this in mind the decrease in consistency of simulation due to growth in number of predicted variable values is compensated by the adequate accounting of features related to the dynamics of production cycle. The model is using the method of consecutive optimization by the sub-periods in planning schedule to shape the program of material provision that is co-related with the dynamics of final product production.

\section{References}

1. Brazhnikov MA. Simulation of Calendar Plans for Assembly Process within the Commercial Machine-Building Production .Bulletin of Samara State Technical University. Series of physical and mathematical science, 2004; 26: 165-173. [in Russian]

2. Buchert T. Multi-criteria Decision Making as a Tool for Sustainable Product Development - Benefits and Obstacles. Procedia CIRP, 2015; 26: 70-75.

3. Burkov VN, Novikov DA. Theory of Active Systems: Status and Perspectives. M.: Synteg, 1999; 26-37. [in Russian]

4. Vlasov RN. Methodic Guidelines for Arrangement and Distribution of Production Programs for the Transport Machine-Building Companies that Follows Regularity in Article Production. Bulletin in Machine-Building, 2008; 9: 77-80. [in Russian] 
5. Domingues AR. Applying Multi-Criteria Decision Analysis to the Life-Cycle Assessment of Vehicles. Journal of Cleaner Production, 2015; 12: 45-48. [in Russian]

6. Faik Bilgili. Business Cycle Co-movements Between Renewables Consumption and Industrial Production: A Continuous Wavelet Coherence Approach. Renewable and Sustainable Energy Reviews, 2015; 52: 325-332.

7. Geraskin MI, Egorova VV. Static Optimization of Production Cycles at Bearing Manufacturing Plants. Bulletin of Samara State Economics University, 2014; 11(121): 53-60. [in Russian]

8. Golinkov YP. 2-Level System in Printing Plant Resource Management. Journal of HEI. Problems in Polygraphy and Printing Business, 2000; 1(2):111-117. [in Russian]

9. Gorlach BA, Saveliev GL. Prediction and Optimization of Material Supply Problems in Conditions of Fluctuating Demand. Bulletin of S.P. Korolev Samara State Aerospace University (National Research University), 2011; 4: 48-57. [in Russian]

10. Gribanova EB. Algorithmic Simulation Models to Manage the Material Resources in a Warehouse. Bulletin of Tomsk Polytechnic University, 2006; 8: 201-206. [in Russian]

11. Zolotarev AN. Reproduction Processes Productivity Growth (at the Example of MachineBuilding Industry): Monograph. Kharkov: "INZHEK" Publishers, 2004; 172 p. [in Russian]

12. Kolodin DO. Updating the Demand Requirements in Equipment in Conditions of a Single Production at the Basis of Production Cycle Model. Actual Innovative Research: Theory and Practice, 2009; 2. URL: http://www.actualresearch.ru. [in Russian]

13. Kolodin DO. Updating the Demand Requirements in Equipment in Conditions of a Single Production at the Basis of Production Cycle Model. Actual Innovative Research: Theory and Practice, 2009; 2. URL: http://www.actualresearch.ru. [in Russian]

14. Kononenko VN. Analysis of Financial Status of the Company (Radid Assessment Method) Management in Russian and Abroad, 2008; 34: 26-37.

15. Materials from website of OOO "Instrument Bearing Plant". URL: http://www.mbfsamara.ru.

16. Materials from website of OAO "Samara Bearing Plant" URL: http://www.spzgroup.ru.

17. Materials from RF RosStat website. URL: http://www.gks.ru.

18. Ogleznev NA. Arrangement and Management of Labour and Production Processes at Machine-Building Industry Plants, Samara: Samara State Aerospace University Publishers, 2007; 416 p. [in Russian]

19. Pakhomov M. Maximum Equipment Load or Minimum Expenses? RISK: Resources, Information, Provision, Competition, 2002; 2: 33-40. [in Russian]

20. Radionov RA. Russian features in Resource and Operating Assets Management. Logistics, 2003; 4: 31-32. [in Russian]

21. Selivanova LA. Evaluation of Companies at the Basis of Intangible Assets Monitoring. Baltic Economic Magazine, 2010; 2(4): 168-179. [in Russian]

22. Sokolov YV. Basic in in Financial Accounting Theory. M: Finance and Statistics, 2005; 496 p. [in Russian]

23. Teplitskiy VA. Securing Stable Financial Development of the Company. Baltic Economic Magazine, 2011; 1(5): 54-59. [in Russian]

24. Tolysbayev BS. Economic and Mathematical Simulation of Agricultural Goods Supply Process. Bulletin of OGU, 2006; 5: 85-88. [in Russian] 


\section{Appendix}

Formula Substantiation (3): This formula comes out of the following correlations and transformations: basic formula to calculate production cycle [22]:

$$
F(u)=\frac{T}{C(u)} \sum_{i=1}^{3} \bar{u}_{i}
$$

where $\bar{u}_{i}$ - average surplus in resources for the period as calculated using formula.

$$
\bar{u}_{i}=\frac{u_{i}^{0}+u_{i}^{T}}{2}, i=1, \ldots, 3 \text {. }
$$

In view of correlations in material, industrial and stock balance reserves [22]

$u_{1}=u_{1}^{T}-u_{1}^{0}+u_{2}, u_{2}=u_{2}^{T}-u_{2}^{0}+u_{3}-P, u_{3}=u_{3}^{T}-u_{3}^{0}+C$,

the average surplus in resources are determined as follows

$\bar{u}_{1}=u_{1}^{0}+\frac{u_{1}-u_{2}}{2}, \bar{u}_{2}=u_{2}^{0}+\frac{u_{2}+P-u_{3}}{2}, \bar{u}_{3}=u_{3}^{0}+\frac{u_{3}-C}{2}$,

where $u_{i}^{T}$ - surplus in resources by the end of the period $\mathrm{T}$.

Substituting (36), (37) and (38) in (35), we get (3).

Argument for Assertion 1: substitute (4) and (6) into (11)

$$
P_{\Sigma}=\sum_{j_{1}=1}^{J_{1}} A_{z j_{1}} u_{1}^{\alpha_{z_{1}}+1}+B_{P}\left(\sum_{j_{1}=1}^{J_{1}} u_{1 j_{1}}\right)^{\beta_{P}}
$$

differentiating (39), we'll write down the required condition for the minimum (11)

$$
P_{\Sigma u_{1 j_{1}}}^{\prime}=A_{z j_{1}}\left(\alpha_{z j_{1}}+1\right) u_{1}^{\alpha_{z j}}+B_{P} \beta_{P}\left(\sum_{j_{1}=1}^{J_{1}} u_{1 j_{1}}\right)^{\beta_{P}-1}=0, j_{1}=1, \ldots, J_{1}
$$

from where we get the correlation (12). Note that the non-negative solution of the equation (12) corresponds to the limit as

$$
\alpha_{z j_{1}}<-1 \text {, }
$$

The sufficient criterion for the minimum 


$$
\begin{aligned}
& P_{\Sigma u_{1 j_{1}}}^{\prime \prime}=A_{z j_{1}} \alpha_{z j_{1}}\left(\alpha_{z j_{1}}+1\right) u_{1}^{\alpha_{z j_{1}}-1}+B_{P} \beta_{P}\left(\beta_{P}-1\right)\left(\sum_{j_{1}=1}^{J_{1}} u_{1 j_{1}}\right)^{\beta_{P}-2}>0, \\
& j_{1}=1, \ldots, J_{1},
\end{aligned}
$$

is completed when

$$
\left\{\begin{array}{l}
\beta_{P}>1 \cap B_{P} \beta_{P}\left(\beta_{P}-1\right)\left(\sum_{j_{1}=1}^{J_{1}} u_{1 j_{1}}\right)^{\beta_{P}-2} \geq A_{z j_{1}}\left|\alpha_{z j_{1}}+1\right| \alpha_{z j_{1}} u_{1 j_{1}}^{\alpha_{z j_{1}}-1}, \forall \alpha_{z j_{1}}<0, \\
\beta_{P} \leq 1 \cap \alpha_{z j_{1}}<-1 \cap A_{z j_{1}}\left(\alpha_{z j_{1}}+1\right) \alpha_{z j_{1}} u_{1 j_{1}}^{\alpha_{z j_{1}}-1}>B_{P} \beta_{P} \mid \beta_{P}-1\left(\left(\sum_{j_{1}=1}^{J_{1}} u_{1 j_{1}}\right)^{\beta_{P}-2},\right.
\end{array}\right.
$$

however, considering (40), condition (41) is observed only with correlation (13).

Argument for Assertion 2: Basing upon the optimality required condition (3):

$$
F_{u_{1}}^{\prime}\left(u_{1}\right)=-\beta_{c} \frac{T u_{\Sigma}^{0}}{B_{c}} u_{1}^{-\beta c-1}+\frac{T}{2 B_{c}}\left(1-\beta_{c}\right) u_{1}^{-\beta c}+\frac{T B_{p}}{2 B_{c}}\left(\beta_{p}-\beta_{c}\right) u_{1}^{\beta p-\beta c-1}=0
$$

we get equation (16) to find parameter $u_{1 F}^{*}$. Sufficient condition for the minimum:

$$
\begin{aligned}
& F_{u_{1}}^{\prime \prime}\left(u_{1}\right)=-\beta_{c}\left(-\beta_{c}-1\right) \frac{T u_{\Sigma}^{0}}{B_{c}} u_{1}^{-\beta c-2}-\frac{T}{2 B_{c}}\left(1-\beta_{c}\right) \beta_{c} u_{1}^{-\beta c-1}+\frac{T B_{p}}{2 B_{c}}\left(\beta_{p}-\beta_{c}\right) \\
& \left(\beta_{p}-\beta_{c}-1\right) u_{1}^{\beta p-\beta c-2}>0
\end{aligned}
$$

we get the following

$$
\beta_{C}\left[2\left(\beta_{C}+1\right) u_{\Sigma}^{0}+\beta_{C}-1\right] u_{1}+B_{P}\left(\beta_{C}-\beta_{P}\right)\left(\beta_{C}+1-\beta_{P}\right) u_{1}^{\beta p}>0 .
$$

As for the actual Companies the order of values $u_{\Sigma}^{0}>>\beta_{C}$, then $2\left(\beta_{C}+1\right) u_{\Sigma}^{0}+\beta_{C}>>1$; that's why sign (43) is under the influence of correlation $\beta_{C}, \beta_{P}$ only. In view of range changes for $\beta_{C}, \beta_{P}$, determined in (4) and (5), the condition (43) in true at

$$
\left\{\begin{array}{l}
\beta_{C}>\beta_{P} \cup\left[\beta_{C}<\beta_{P} \cap \beta_{C}+1<\beta_{P}\right] \forall u_{1 F 1}^{*} \geq 0, \\
\beta_{C}<\beta_{P} \cap \beta_{C}+1>\beta_{P} \cap \varphi\left(\beta_{C}, \beta_{P}, u_{\Sigma}^{0}, u_{1}\right)>0,
\end{array}\right.
$$

where 


$$
\begin{aligned}
& \varphi\left(\beta_{C}, \beta_{P}, u_{\Sigma}^{0}, u_{1}\right)=\beta_{C}\left[2\left(\beta_{C}+1\right) u_{\Sigma}^{0}+\beta_{C}-1\right] u_{1}+ \\
& +B_{P}\left|\beta_{C}-\beta_{P}\right|\left(\beta_{C}+1-\beta_{P}\right) u_{1}^{\beta p}
\end{aligned}
$$

In view of (15) the condition (440) is as follows (17).

Argument for Assertion 3: let's have the formula of Lagrange for the (9) with restriction (10)

$$
L=F\left(u_{1}\right)+\lambda_{1}\left(u_{1}^{\min }(N)-u_{1}\right)+\lambda_{2}\left(u_{1}^{\max }(N)-u_{1}\right)+\lambda_{3}\left(u_{1}^{\min }(z)-u_{1}\right),
$$

and with differentiation we'll get the system of optimality required conditions

$$
\begin{aligned}
& \frac{\partial L}{\partial u_{1}}=\frac{\partial F\left(u_{1}\right)}{\partial u_{1}}-\lambda_{1}-\lambda_{2}-\lambda_{3}=0, \\
& \frac{\partial L}{\partial \lambda_{1}}=u_{1}^{\min }(N)-u_{1} \leq 0, \frac{\partial L}{\partial \lambda_{2}}=u_{1}^{\max }(N)-u_{1} \geq 0, \frac{\partial L}{\partial \lambda_{3}}=u_{1}^{\min }(z)-u_{1} \leq 0,
\end{aligned}
$$

While computing (47) as rigid imparity for the solution of the system (46), (47) will be the vector of Lagrange multipliers $\lambda_{1}=\lambda_{2}=\lambda_{3}=0$, respectively, from (46) the optimality required conditions are coming out as $\frac{\partial F\left(u_{1}\right)}{\partial u_{1}}=0$, presented in form (16) to define the optimum criteria without any restrictions in $u_{1 F}^{*}$, and at this $u_{1}^{*}=u_{1 F}^{*}$. In case we complete any of the conditions (47) as rigid congruence by system solutions (46), (47) will be presenting the parameter of management, satisfying the conditions

$$
u_{1}=u_{1}^{\min }(N) \cup u_{1}=u_{1}^{\max }(N) \cup u_{1}=u_{1}^{\min }(z),
$$

That will be formally presented as (23). The sufficient condition of extremum in task (9) with restriction (10) is completed, if the sufficient condition (17) defines the range of $u_{1 F}^{*} \in U$. 